\title{
Efficacy and safety of erlotinib in the treatment of metastatic non-small-cell lung cancer
}

\author{
This article was published in the following Dove Press journal: \\ Lung Cancer:Targets and Therapy \\ 20 December 2010 \\ Number of times this article has been viewed
}

\author{
Serena Ricciardi' \\ Silverio Tomao ${ }^{2}$ \\ Filippo de Marinis' \\ 'Thoracic-Oncology Unit Ist, \\ Lung Diseases Department, San \\ Camillo-Forlanini High Specialization \\ Hospitals, Rome, Italy; ${ }^{2}$ Sperimental \\ Medicine Department, La Sapienza \\ University, Rome, Italy
}

\begin{abstract}
Lung cancer is the leading cause of cancer deaths worldwide. Targeting the epidermal growth factor receptor (EGFR) has played a central role in advancing non-small-cell lung cancer (NSCLC) research, treatment, and patient outcome over the last few years. Erlotinib is a human epidermal growth factor receptor Type 1/EGFR tyrosine kinase inhibitor. Erlotinib monotherapy is indicated for the treatment of patients with locally advanced or metastatic NSCLC after the failure of at least one prior chemotherapy regimen. We present the results of phase I, II, III, and IV trials. Erlotinib monotherapy has shown a significant improvement in median survival, quality of life, and related symptoms in an unselected population of patients in second- and third-line therapy as a single agent. Most commonly reported erlotinib-related adverse effects were rash and diarrhea. In general, patients with adenocarcinoma histology, female gender, Asian ethnicity, and nonsmokers have a better response when treated with erlotinib. Ongoing trials seek to improve therapy with this agent in monotherapy or in combination regimens and better understanding of predictors of benefit with therapy.
\end{abstract}

Keywords: erlotinib, NSCLC, targeted therapy

\section{Introduction}

Lung cancer is the leading cause of cancer deaths worldwide. Approximately 400,000 new cases of lung cancer are diagnosed every year in Europe; lung carcinoma represents about $22 \%$ of all newly diagnosed cancers in men. ${ }^{1}$

Progress has been made in the primary prevention of lung cancer and in the clinical management of early stage disease by establishing comprehensive, multimodality treatment regimens. The prognosis for advanced stage disease, however, has improved modestly in the past 20 years. With an overall 5 -year survival rate of only $15 \%$, the treatment of lung cancer clearly remains a major clinical challenge. ${ }^{2}$ Patients are often diagnosed at an advanced stage of the disease, and approximately $85 \%$ of patients die because of their disease within 1 year, with only $1 \%$ of patients surviving 5 years. Patients having stage IIIB/IV non-small-cell lung cancer (NSCLC) are not considered to be candidates for curative resection surgery or radiation, and radiation therapy is primarily used as palliative treatment in advanced stages of NSCLC. Surgical results in earlier stages of NSCLC are poor (about $40 \%$ of recurrence in stages I and II) when compared with that of the other tumor types. ${ }^{3}$

Chemotherapy is now well established as the recommended treatment of advanced NSCLC. The current globally accepted standard of treatment for advanced NSCLC is two-drug platinum-based therapy with new agents such as vinorelbine, taxanes, 
gemcitabine, or pemetrexed in patients with nonsquamous histology. For selected nonsquamous NSCLC, bevacizumab with paclitaxel-carboplatin represents a new option. ${ }^{4}$

One innovative therapeutic cancer strategy is the introduction of biological agents that target specific intracellular pathways related to the distinctive properties of cancer cells. Among these agents, epidermal growth factor receptor (EGFR)-targeting agents have received particular attention in lung cancer.

In recent years, the major progresses in understanding cancer biology and oncogenesis mechanisms have provided for the development of several potential molecular targets for NSCLC treatment. Targeting the EGFR has played a central role in advancing NSCLC research, treatment, and patient outcome over the last few years. Many EGFR blockers, including monoclonal antibodies to the receptor and small-molecule tyrosine kinase inhibitors (TKIs), have been evaluated. ${ }^{5}$

This article describes the development of erlotinib, focusing on the clinical data reported in the treatment of NSCLC.

\section{Mechanism of action and pharmacodynamics}

Erlotinib monotherapy is indicated for the treatment of patients with locally advanced or metastatic NSCLC after the failure of at least one prior chemotherapy regimen. Erlotinib was approved by the US Food and Drug Administration (FDA) in November 2004 and by the European Medicinal Evaluation Agency (EMEA) in October 2005.

Erlotinib (Tarceva ${ }^{\circledR}$; Genentech, OSI Pharmaceuticals, Roche) is a human epidermal growth factor receptor Type 1 (HER1)/EGFR TKI. The mechanism of clinical antitumor action of erlotinib is not completely characterized. Erlotinib inhibits the intracellular phosphorylation of tyrosine kinase associated with the EGFR. The EGFR is a member of the ErbB family of cell membrane receptors that are important mediators of cell growth, differentiation, and survival. The ErbB family also includes ErbB-2/Neu/HER2, ErbB-3/ HER3, and ErbB-4/HER4.,

Specificity of inhibition with regard to other tyrosine kinase receptors has not been fully characterized. EGFR is expressed on the cell surface of both normal cells and cancer cells. The EGFR is frequently overexpressed in various epithelial tumors, including NSCLC (40\%-80\%), colorectal cancer $(72 \%-82 \%)$, head and neck cancer $(95 \%-100 \%)$, breast cancer ( $84 \%-91 \%)$, and renal cell cancer $(50 \%-90 \%)$. The blockade of EGFR signaling in cancer cells not only inhibits cell proliferation, but also causes other effects that could be relevant in the clinical setting, including the induction of apoptosis, antiangiogenesis through the inhibition of angiogenic growth factor production, inhibition of invasion and metastasis, and potentiation of the antitumor activity of cytotoxic drugs and radiotherapy. ${ }^{8}$

About $60 \%$ of erlotinib is absorbed after oral administration, and its bioavailability is substantially increased by food to almost $100 \%$. Its half-life is about 36 hours, and it is cleared predominantly by CYP3A4 metabolism and to a lesser extent by CYP1A2.

In vitro assays of cytochrome $\mathrm{P} 450$ metabolism showed that erlotinib is metabolized primarily by CYP3A4 and to a lesser extent by CYP1A2 and the extrahepatic isoform CYP1A1. Following a $100 \mathrm{mg}$ oral dose, $91 \%$ of the dose was recovered: $83 \%$ in feces ( $1 \%$ of the dose as intact parent compound) and $8 \%$ in urine $(0.3 \%$ of the dose as intact parent compound). Cotreatment with the potent CYP3A4 inhibitor ketoconazole increased erlotinib AUC by two-thirds. Pre- or cotreatment with a CYP3A4 inducer (rifampicin) or antiepiletics (phenytoin, carbamazepine, and barbiturates) increased erlotinib clearance by threefold and reduced AUC by two-thirds. ${ }^{9}$

\section{Phase I trials}

Phase I studies were undertaken to determine the maximum tolerated dose (MTD) of erlotinib, its main toxicities, and its pharmacokinetic behavior to recommend a dose for subsequent studies..$^{10}$ Results of these investigations showed that erlotinib had dose-independent pharmacokinetics and that daily dosing of erlotinib did not result in drug accumulation.

The initial phase I trial in solid tumors ${ }^{11}$ evaluated different doses $(25,50,100,150$, and $200 \mathrm{mg})$ and schedules (days 1-3 weekly for 3 weeks every 28 days; daily for 3 weeks every 28 days; and daily uninterrupted) of erlotinib, and $150 \mathrm{mg} /$ day erlotinib was determined to be the MTD at which biologically relevant plasma levels were achieved. The most common toxicities associated with erlotinib in phase I trials were dose-dependent acneiform rash and diarrhea. A similar rash was observed with other TKIs. Diarrhea is also a common side effect seen with other TKIs, leading to the speculation that these symptoms are a result of inhibition of the EGFR. The histological characteristics of the rash caused by erlotinib differ from that of typical acne. The cutaneous toxicities were mostly on the face and upper trunk and of a pustular acneiform type. The rash appeared 1-2 weeks after the initiation of therapy and subsided by week 4 without the interruption of the erlotinib. Other less-common adverse reactions were mucositis, nausea, vomiting, and 
headache. The pharmacokinetics of erlotinib was not dose dependent, and there was no drug accumulation with the continuous daily dosing.

The weekly regimen was explored further in patients with advanced stage NSCLC with dose escalation of 1200, 1600, and $2000 \mathrm{mg}$, but was discontinued due to a low response rate (relative risk [RR]; 5\%). ${ }^{12}$

\section{Phase II trials}

Perez-Soler et $\mathrm{al}^{13}$ have conducted a phase II clinical trial to evaluate the effect of erlotinib at a dose of $150 \mathrm{mg} /$ day in EGFR-positive (determined by immunohistochemistry [IHC]) advanced refractory NSCLC.

Fifty-seven patients were enrolled. Median survival time (MST) was 8.4 months (95\% confidence interval [CI], 4.8-13.9 months), the 1-year survival rate was $40 \%$, and median progression-free survival (PFS) was 9 weeks (95\% CI, 8-15 weeks). The most common side effects were rash (67\%) and diarrhea (56\%). Patients who developed a rash survived longer (1.5 months) than those who do not have a rash, and the MST was longer for those with grade 2 or 3 rash (19.6 months) than for those with grade 1 rash ( 8.5 months). This positive correlation suggests that rash may be a marker of HER1/EGFR kinase inhibition with the use of erlotinib.

Erlotinib as a single agent has also been tested as firstline treatment in advanced NSCLC patients. ${ }^{14}$ Fifty-three chemotherapy-naive patients with stage IIIB/IV NSCLC were enrolled to receive erlotinib.

The overall rate of nonprogression at 6 weeks was $52.8 \%$ ( 28 out of 53 patients). Tumor RR was $22.7 \%$, with one complete response (CR), 11 partial response (PR), and 16 cases of stable disease (SD). The median duration of tumor response was 333 days, median overall survival (OS) was 391 days, and median time to progression (TTP) was 84 days. Responses were more common in women, adenocarcinoma histology, and in nonsmoker patients.

Five patients were reported to have a grade 3 adverse event (AE). In 2007, Jackman et $\mathrm{al}^{15}$ reported data from a phase II clinical trial evaluating erlotinib as first-line monotherapy in patients $>70$ years of age, chemotherapy-naive, with stage III/IV disease treated with erlotinib at $150 \mathrm{mg} / \mathrm{m}^{2}$. The MST was 10.9 months. The 1- and 2-year survival rates were $46 \%$ and $19 \%$, respectively. Eighteen patients experienced a grade 3 AE, most commonly rash (five patients) and interstitial pneumonitis (three patients). The toxicity of erlotinib in this population compares favorably with that seen in other studies performed in patients with NSCLC $<70$ years of age.

Phase II trials ${ }^{16}$ evaluating the effects of erlotinib in untreated patients as first-line treatment in advanced NSCLC and a performance status (PS) of 2 were presented at the 2007 Annual Meeting of the American Society of Clinical Oncology.

The treatment with erlotinib was well tolerated in PS-2 patients who are generally less likely to tolerate standard-dose platinum-based doublet chemotherapy.

In the same meeting, Jackman et a ${ }^{17}$ presented a phase II trial evaluating the effects of erlotinib in 49 chemotherapynaive women with advanced adenocarcinoma NSCLC. Preliminary data reported 34\% RRs, 28\% SDs, and PFS and MST were 5.8 and 23.2 months, respectively.

\section{Phase III trials}

The efficacy of erlotinib has been tested in both monotherapy and combined with chemotherapy in several phase III clinical trials.

\section{Monotherapy}

The BR.21 study was a phase III randomized, double-blind, placebo-controlled trial assessing the efficacy of erlotinib treatment of patients with advanced and chemotherapyrefractory NSCLC. ${ }^{18}$ A total of 731 patients were randomly assigned in a 2:1 ratio to receive either erlotinib or placebo.

Patients were required to have received one or more previous lines of chemotherapy. At the time of the study, pemetrexed was not available, and there was concern about the toxicity and effectiveness of further chemotherapy after the failure of standard chemotherapy in some patients; therefore, it was reasonable to compare erlotinib with placebo. The primary end point of the study was OS (Table 1). RR was $8.9 \%$ in the erlonitib group and less than $1 \%$ in the placebo group; median response duration was 7.9 and 3.7 months, respectively. Median survival was 6.7 months with erlotinib and 4.7 months with placebo $(P<0.001)$. One-year survival was $31 \%$ with erlotinib and $21 \%$ with placebo. The objective responses (ORs) were more frequent in women $(14 \%$ vs $6 \% ; P<0.0065)$ and in patients with adenocarcinoma, as compared with other histotypes (14\% vs $4.1 \% ; P<0.0001)$ and in patients without a smoking history ( $25 \%$ vs $4 \% ; P<0.0001$; Table 2$)$.

Table I Survival results from BR.2I: a phase III randomized, double-blind, placebo-controlled trial assessing the efficacy of erlotinib treatment of patients with advanced and chemotherapyrefractory NSCLC ${ }^{18}$

\begin{tabular}{llll}
\hline & $\begin{array}{l}\text { Erlotinib } \\
(\boldsymbol{n}=\mathbf{4 8 8})\end{array}$ & $\begin{array}{l}\text { Placebo } \\
(\boldsymbol{n}=\mathbf{2 4 3 )}\end{array}$ & $\boldsymbol{P}$ value \\
\hline Progression-free survival (months) & 2.2 & 1.8 & $<0.00 \mathrm{I}$ \\
Overall survival (months) & 6.7 & 4.7 & $<0.00 \mathrm{I}$ \\
One-year survival (\%) & $3 \mathrm{I}$ & 22 & $\mathrm{NA}$ \\
\hline
\end{tabular}


The BR.21 was the first clinical trial in which a novel targeted agent, such as erlotinib, significantly improved the survival of chemotherapy-refractory advanced NSCLC patients.

The most common toxicities seen in this trial were diarrhea and rash as was seen in the previous studies. Dose reductions were carried out in $12 \%$ of patients due to rash and 5\% for diarrhea. Erlotinib was discontinued only in $5 \%$ of patients due to toxicity. In contrast to most cytotoxic agents, grade 3 or 4 neutropenia was not reported with erlotinib. In the BR.21 study, pulmonary infiltrates and pneumonitis (3\%) were seen equally in the erlotinib vs placebo arm. One patient died on each arm due to pneumonitis, which was most likely related to the underlying lung cancer and not the drug.

The quality-of-life analysis supports the true palliative benefit of erlotinib in improving not only survival but also the median time to deterioration for all three principal symptoms related to the disease (4.9 vs 3.7 months for cough $[P=0.04]$; 4.7 vs 2.9 months for dyspnea $[P=0.04]$; and 2.8 vs 1.9 months for pain $[P=0.03]) .{ }^{19}$

\section{Chemotherapy combination}

Erlotinib in combination with chemotherapy for the firstline treatment of NSCLC has been evaluated in two large multicenter, randomized, placebo-controlled clinical trials (Table 3).

The TRIBUTE trial ${ }^{20}$ was conducted in the treatment of naive patients with advanced or metastatic NSCLC who were treated with chemotherapy plus erlotinib vs placebo.

The clinical trial compared the OS of patients with NSCLC receiving either erlotinib in combination with paclitaxel and carboplatin or paclitaxel plus carboplatin alone.

This prospective, placebo-controlled trial, randomized patients with previously untreated advanced NSCLC (stage IIIB/4) to receive erlotinib at a dose of $150 \mathrm{mg} /$ day or placebo with six cycles of carboplatin plus paclitaxel followed by maintenance monotherapy. The primary end point was OS.

A total of 1,059 patients were randomized. There was no difference in patients treated with erlotinib and carboplatin/

Table 2 Response rate to erlotinib in different patient subgroups in BR.2I: gender, histologies, race, and smoker ${ }^{18}$

\begin{tabular}{lll}
\hline & Response rate (\%) & $\boldsymbol{P}$ \\
\hline Women vs men & 14 vs 6 & 0.006 \\
Adenocarcinoma vs other histologies & 14 vs 4 & $<0.001$ \\
Asian vs non-Asian & 19 vs 8 & 0.02 \\
Nonsmoker vs current/former & 25 vs 4 & $<0.00$ I \\
\hline
\end{tabular}

paclitaxel when compared with those treated with carboplatin/paclitaxel alone: OS was 10.8 months with erlotinib vs 10.6 months with placebo ( $P=0.95$; hazard ratio [HR], 0.99), OR was 5.5 months with erlotinib vs 5.0 months with placebo $(P=0.32$; HR, 0.85), and median TTP was 5.1 months with erlotinib vs 4.9 months with placebo ( $P=0.36$; HR, 0.94).

Rash and diarrhea were more common in the erlotinib group (47.7\% with erlotinib vs $43.2 \%$ with placebo). This study concluded that erlotinib combined with carboplatin/ paclitaxel chemotherapy does not confer a survival advantage over carboplatin/paclitaxel alone in previously untreated advanced NSCLC.

Another placebo-controlled randomized study (TALENT) was reported in the treatment of naive unresectable stage III or IV NSCLC. ${ }^{21}$ In this study, patients received six cycles of cisplatin $\left(80 \mathrm{mg} / \mathrm{m}^{2}\right.$ day 1$)$ and gemcitabine $\left(1250 \mathrm{mg} / \mathrm{m}^{2}\right.$ days 1,8$)$ plus erlotinib (150 mg daily) or placebo with responding patients who continued to receive erlotinib until progression.

There was no difference in RRs, OS, TTP, and time to symptom progression among the 1,172 patients enrolled.

The exception to this was a survival benefit seen in a subset analysis of nonsmokers. Based on these two randomized trials, erlotinib has no role in first-line therapy when given concurrently with chemotherapy in an unselected patient population.

\section{Phase IV trial}

Tarceva Survival Lung Cancer Treatment (TRUST) study is an open-label, nonrandomized, multicenter, phase IV trial in patients with advanced NSCLC who have failed standard chemotherapy, cannot receive other systemic anticancer therapy, are not medically suitable for chemotherapy, or are ineligible for other clinical trials with erlotinib. The trial was designed to allow access to erlotinib monotherapy for suitable patients in countries where the drug had not been licensed yet. Almost 7,000 patients have been enrolled for the TRUST study at 549 centers in 52 countries worldwide. $^{22}$

In February 2008, data were available for 4,002 European patients. ${ }^{23}$ Twenty-three patients $(<1 \%)$ had CR, 281 (9\%) had PR, and 1,864 (58\%) had SD. Median PFS was 12.3 weeks (95\% CI, 11.9-12.9); 1-year survival was 32\%; and median OS was 6.7 months. Degree of rash was correlated with survival. Subanalyses showed that patients who were female, PS 0 or 1, nonsmokers, or who had adenocarcinoma had prolonged PFS. Degree of rash was correlated with survival. 
Table 3 Erlotinib in combination with chemotherapy for the first-line treatment of NSCLC

\begin{tabular}{|c|c|c|c|c|c|}
\hline Study & No of patients & Regimen & $\begin{array}{l}\text { Median survival } \\
\text { (months) }\end{array}$ & $\mathbf{R R}$ & $\begin{array}{l}\text { Overall } \\
\text { survival (\%) }\end{array}$ \\
\hline \multirow[t]{2}{*}{ TALENT $^{21}$} & 1,172 & $\mathrm{CT}+$ placebo & 10.2 vs 9.9 & NR & NR \\
\hline & & $\mathrm{CT}+$ erlotinib & $P=N S$ & & \\
\hline \multirow[t]{2}{*}{ TRIBUTEE ${ }^{20}$} & 533 & $\mathrm{CT}+$ placebo & 10.5 vs 10.6 & 19.3 vs 21.5 & 44 vs 47 \\
\hline & & $\mathrm{CT}+$ erlotinib & $P=0.95$ & $P=0.36$ & \\
\hline
\end{tabular}

Abbreviations: $\mathrm{RR}$, relative risk; $\mathrm{CT}$, cisplatin.

Recently, Tiseo et $\mathrm{al}^{24}$ reported the interim analysis from the Italian experience. At the time of this analysis, data from 651 patients were available. Erlotinib was administered as first-, second-, third-, or other-line therapy. RR was $9 \%$, with a disease-control rate of $63 \%$. Median PFS was 15 weeks and was longer in females $(P<0.001)$, patients with adenocarcinoma $(P=0.008)$, nonsmokers $(P<0.001)$, and patients who experienced skin toxicity $(P<0.001)$. Safety data were available for 609 patients, $35 \%$ of whom had at least one $\mathrm{AE}$, but only $4 \%$ of patients discontinued treatment due to erlotinib-related AEs. The results of TRUST study achieved using erlotinib in a large unselected Italian population with advanced NSCLC confirm the activity and favorable tolerability, which are similar to that achieved in the BR.21 trial.

\section{Combination with other targeted-agents}

Many novel targeted agents are currently in clinical trials in combination with erlotinib; bevacizumab was combined with erlotinib in a phase I/II study in patients with relapsed nonsquamous NCSLC..$^{25}$

No dose-limiting toxicity was reported in the phase I portion, and 34 patients were treated at the phase II doses with erlotinib $150 \mathrm{mg}$ daily and bevacizumab $15 \mathrm{mg} / \mathrm{kg}$ every 21 days. The most common toxicities were diarrhea, rash, hematuria, and proteinuria, and there were no treatmentrelated deaths. There was a $20 \% \mathrm{PR}$ and a $65 \%$ SD with a median OS of 12.6 months and a PFS of 6.2 months. Nine tumors were tested for EGFR mutations in exons 19-21 and 23, and only two had the mutation (1 PR and $1 \mathrm{SD}$ ).

In 2006, Fehrenbacher et al ${ }^{26}$ published data about a phase II, multicenter, randomized clinical trial. The study evaluated the efficacy and safety of bevacizumab in combination with chemotherapy or erlotinib. One hundred twenty advanced nonsquamous NSCLC patients, previously treated with a platinum-based regimen, were randomized to receive docetaxel or pemetrexed plus placebo (arm 1), docetaxel or pemetrexed plus bevacizumab (arm 2), or bevacizumab plus erlotinib (arm 3). The overall rate of nonprogression was
$39 \%$ in arm 1, compared with $52.5 \%$ in arm 2 and $51.3 \%$ in arm 3. The percentage of patients free from progression at 6 months was $21.5 \%$ in arm 1 , vs $30.5 \%$ in arm 2 , and $33.6 \%$ in arm 3. The 6-month survival rate was $62.4 \%$ in arm 1 , vs $72.1 \%$ in arm 2 , and $78.3 \%$ in arm 3 .

Confirmation of these exciting preliminary results is being sought in two international phase III trials: ATLAS, ${ }^{27}$ and BETA. The ATLAS trial $(N=1150)$ is a randomized, double-blind, placebo-controlled phase IIIB trial that compares bevacizumab with or without erlotinib after the completion of first-line chemotherapy with bevacizumab for advanced nonsquamous NSCLC.

Recently, Miller et $\mathrm{al}^{27}$ has presented preliminary data: the median PFS was 3.75 months vs 4.76 months, respectively, for patients with only bevacizumab $(n=370)$ and for group with bevacizumab and erlotinib $(n=373)$. The PFS at 6 months was 28.4 vs 40.3 , respectively, for bevacizumab vs bevacizumab + erlotinib. The data concerning the survival will be available to the ASCO 2010.

The BETA trial ${ }^{28}(N=650)$ randomized patients requiring second-line therapy to erlotinib with or without bevacizumab. The preliminary not-yet-published data showed no advantage for the combination arm.

\section{Predictors of response Clinical predictors}

There is some tendency to consider that erlotinib is only effective in certain subgroups of patients, such as women or Asians, or among patients with adenocarcinoma. ${ }^{29}$ Analysis of data from subgroups included in the BR.21 trial showed that OS is similar to women and men (HR, 0.8 for both populations). ${ }^{18}$ Likewise, survival is similar among patients with adenocarcinoma and epidermoid carcinoma (HR, 0.7 and 0.67 , respectively).

The results of Asian patients and other ethnicities are similar (HR, 0.6 and 0.8 , respectively). The most important group is of male smokers with epidermoid carcinoma. In this population, HR in the erlotinib group $(n=100)$ is 0.66 , and MST was 5.5 months, as compared to 3.4 months in the placebo group $(n=57) .{ }^{30}$ 
A subgroup analysis of the TRIBUTE trial showed that the addition of erlotinib to carboplatin or paclitaxel prolonged the survival in patients who never smoked (MST, 22.5 vs 10.1 months; HR, $0.49 ; 95 \%$ CI, $0.28-0.85 ; P=0.01)$. The RR to chemotherapy with erlotinib was also higher in the nonsmokers when compared with that to chemotherapy alone (21 [30\%] of $69 ; 95 \%$ CI, $20 \%-43 \%$ vs 5 [11\%] out of 44 ; $95 \% \mathrm{CI}, 4 \%-25 \% ; P=0.02){ }^{20}$

Dermatologic toxicities are the most common AEs associated with EGFR inhibitors, which occur in $>50 \%$ of patients who receive treatment. The rash is dose dependent. ${ }^{31}$

Furthermore, in the phase III TRIBUTE and TALENT trials, a subanalysis showed that patients who developed rash survived longer than those who did not. ${ }^{32}$ Finally, it was recently shown that susceptibility to rash and clinical activity of EGFR-targeting agents could be linked to polymorphic variations in the EGFR gene. ${ }^{33}$ The relationship between the development of rash and survival is currently being evaluated further, and the results should help to guide the use of EGFR-targeted therapy.

\section{Molecular predictors}

The degree of EGFR expression can be evaluated by IHC and the EGFR gene copy number by fluorescent in situ hybridization (FISH). EGFR expression determined by IHC has been the first biological marker to be investigated as a possible molecular predictor of response to treatment with TKIs.

Expression levels did not correlate with survival, stage, age, gender, or smoking history. Well-differentiated tumors had a higher level of EGFR expression when compared with poorly differentiated tumors. ${ }^{34}$

In 2006, Miller et $\mathrm{al}^{35}$ published data from prospective phase II trial about patients with bronchioloalveolar carcinoma (BAC). Gene amplification in combination with EGFR mutation (exons 19 and 21) was shown to be a strong predictor of response to erlotinib. Patients with both EGFRactivating mutations and gene amplification had a 90\% RR and a median OS of 35 months. In comparison, patients with no mutations and no gene amplification had a $4 \%$ RR and median OS of 15 months.

In the BR.21 study, EGFR expression in the erlotinibtreated group was associated with a better response without a survival advantage. ${ }^{36}$ Thus, EGFR expression by IHC alone does not seem to be useful in predicting survival after erlotinib therapy. The FISH analysis from BR.21, however, did show a striking benefit in survival for the EGFR-positive patients vs the EGFR-negative patients $(P=0.002)$.
In a retrospective analysis of the TRIBUTE trial, FISH analysis was successfully performed on 245 patients. In EGFR-positive patients, OS was similar between those treated with chemotherapy plus erlotinib and those treated with chemotherapy alone. However, EGFR-positive patients in the combination group arm experienced a decrease in $\mathrm{RR}$ compared with those in the $\mathrm{CP}$-alone arm, and had a marginally significant longer TTP (HR, 0.59; 95\% CI, 0.35-0.99). The benefit in TTP appeared after approximately 6 months, during the maintenance period of the trial. ${ }^{37}$

It has been argued that only patients with certain specific mutations respond to erlotinib. The most common mutations are in a frame deletion in exon 19 around codons 746-750 ( $45 \%-50 \%$ of all somatic EGFR mutations) and a missense mutation leading to leucine-to-arginine substitution at codon 858 (L858R) in exon 21 (35\%-45\% of mutations). ${ }^{38}$ Somatic mutations in the EGFR gene are most frequently detected in a subpopulation of NSCLC patients with characteristics associated with a better treatment outcome, including adenocarcinomas histology and in particular, BAC, nonsmokers, patients of Asian ethnicity, and females. ${ }^{39,40}$

In the BR.21 mutational analysis study, 40 of 177 (23\%) samples were positive for mutations in exons $18-21 .{ }^{36}$ EGFR mutations were found at varying levels in the following subgroups: males (22\%), females (24\%), Asians (50\%), non-Asians ( $21 \%$ ), nonsmokers ( $31 \%$ ), and adenocarcinoma $(28 \%)$. In this trial, surprisingly, the presence of mutations did not correlate with response or survival even in patients with classic exon 19 or 21 mutations. There was a trend toward better RR in those with mutations, but this was not of statistical significance.

In the TRIBUTE study, ${ }^{41}$ patients with EGFR mutations also showed an increase in response to treatment (38\%), when compared with wild-type cases $(23 \% ; P=0.01)$, and a prolonged survival that was independent of treatment ( $8 \mathrm{vs}$ 5 months for mutation-positive and mutation-negative groups, respectively; $P<0.001)$. Similar results have emerged from retrospective molecular analyses from the TALENT trial. ${ }^{42}$

\section{Adverse effects and compliance}

Erlotinib is not significantly associated with hematological adverse effects. ${ }^{18}$ Most commonly reported erlotinib-related adverse effects were rash and diarrhea. Diarrhea is not a major problem, because it may be treated with standard therapies. Rash was found in around 1 out of 20 patients receiving erlotinib.

Because the EGFR is expressed in the skin and in particular, in undifferentiated keratinocytes of the basal layers of 
the epidermis and the outer root sheath of the hair follicle, ${ }^{43}$ the reported side effects are acneiform eruption, xerosis, telangiectasia, hyperpigmentation, fissures, and hair and nail changes. It has been suggested that the variability in skin toxicity may be related to pharmacogenetic and pharmacodynamic differences or pharmacogenetic, heterogenetic, and EGFR polymorphisms among patients. ${ }^{44}$

Data from 485 patients enrolled in the BR.21 study ${ }^{18}$ showed that skin rash occurred in $79 \%$ of the patient population. The severity of rash was mild to moderate. Grades 3 and 4 occurred in $8 \%$ and $<1 \%$, respectively, of erlotinibtreated patients.

The rash seems to be dose dependent, with higher incidence and more severe rash at higher dose levels. ${ }^{13}$ In general, the rash manifests within a week after treatment with EGFR inhibitors and reach a maximum level after 2-3 weeks. The reaction is reversible, usually with complete resolution within 4 weeks of withdrawal from treatment. Data from several clinical trials with HER1/EGFR-targeted agents show a positive correlation between rash and response and/or survival. ${ }^{45}$ These findings suggest that rash may be a surrogate marker of efficacy. However, this raises the question of why about one-third of patients do not develop skin toxicity, even at the MTD.

Perez-Soler et $\mathrm{al}^{46}$ has performed a multivariate analysis of factors affecting survival, including skin toxicity, and found the rash to be a significant predictor of survival. Prospective randomized studies investigating the response-rash relationship are needed to confirm the relationship between rash and clinical benefit.

\section{Future directions and conclusions}

Erlotinib has a clear role in second- or third-line treatment of NSCLC. The decision of when to administer (either as second or third line) can be challenging. Certain patients, such as nonsmokers and those with known EGFR mutations, will obviously be offered second-line therapy with the drug. However, for those patients without favorable clinical or molecular predictors of response, it is difficult to determine whether erlotinib is as efficacious as either docetaxel or pemetrexed. ${ }^{47}$ The ongoing TITAN trial will hopefully answer this question. This phase III trial randomizes patients to receive either erlotinib or docetaxel or pemetrexed.

Major areas of clinical research are the assessment of erlotinib in adjuvant treatment, in the first-line therapy of advanced disease, and in combination and/or sequence with cytotoxic treatments and/or other molecular-targeted agents. ${ }^{32}$

An ongoing adjuvant trial, RADIANT, is open worldwide to patients with stage I-IIIA resected NSCLC who have evidence of EGFR overexpression by either IHC or FISH. Eligible patients will be randomized to either 2 years of erlotinib or observation, after the completion of adjuvant chemotherapy at the discretion of the treating physician.

The efficacy of erlotinib in the first-line setting in patients with advanced NSCLC and activating EGFR mutations was demonstrated in a phase II trial of the Spanish Lung Cancer Group. ${ }^{48}$ Screening of 2,507 patients detected activating EGFR mutations in 358 patients, of whom 217 participated in the study. TTP was 14 months and median OS was 27 months. To determine the predictive value of activating EGFR mutations in first-line therapy with erlotinib, the Phase III trial EURTAC was initiated, in which patients with proven activating EGFR mutations receive erlotinib or platinumbased doublet chemotherapy in the first-line setting.

The Tarceva or Chemotherapy (TORCH) study ${ }^{49}$ is a Phase III randomized, multicenter trial based on a noninferiority survival comparison between an experimental strategy including first-line erlotinib followed at progression by chemotherapy with cisplatin and gemcitabine (PG) and a standard arm consisting of first-line PG chemotherapy followed at progression by erlotinib. Moreover, this trial will allow the evaluation of the relationship between molecular predictors, such as EGFR and K-ras mutational status and erlotinib treatment response. The study design is based on a noninferiority survival comparison with about 900 patients.

In November 2009, the enlistment of the patients has been interrupted because of the experimental arm inferiority showed by interim analysis.

Recently, Cappuzzo et a ${ }^{50}$ presented data from the Sequential Tarceva in Unresectable NSCLC (SATURN) trial a double-blind, randomized, phase III study of maintenance erlotinib vs placebo following nonprogression with first-line platinum-based chemotherapy in patients with advanced NSCLC. The final results of the study found that the median OS for patients treated with erlotinib after chemotherapy was about 12 months vs 11 months for patients who were not treated with the EGFR inhibitor. The difference represented a $19 \%$ reduction in the risk of cancer progression $(P=0.0088)$. The use of erlotinib following chemotherapy resulted in a $29 \%$ reduction in the risk of disease progression $(P<0.0001)$. Subanalyses showed that patients who had mutation or who had adenocarcinoma had prolonged PFS. Despite the positive data, the FDA Advisory Panel is negatively express around the approval of erlotinib in firstline maintenance therapy in patients with advanced NSCLC. Actually, ongoing trials seek to improve therapy with this agent in monotherapy or in combination regimens and better 
Table 4 Ongoing phase I, II, and III trials with erlotinib ${ }^{\mathrm{a}}$

\begin{tabular}{|c|c|c|}
\hline Study phase & Treatment & Primary end point \\
\hline II & $\begin{array}{l}\text { Erlotinib plus RI507 in patients with IIIB/IV NSCLC with progressive disease after clinical } \\
\text { benefit with erlotinib }\end{array}$ & PFS \\
\hline I & Erlotinib plus Sorafenib in patients with pretreated unresectable stage III or IV NSCLC & Safety \\
\hline III & Erlotinib vs docetaxel or pemetrexed & OS \\
\hline II & Erlotinib in combination with chemoradiation in patients with stage IIIA/B & $\begin{array}{l}\text { Time to disease } \\
\text { progression }\end{array}$ \\
\hline II & Erlotinib with concurrent whole brain radiation therapy in patients with brain metastases & OS \\
\hline II & $\begin{array}{l}\text { Erlotinib plus docetaxel in patients with advanced or metastatic NSCLC after failure } \\
\text { of one chemotherapy regimen }\end{array}$ & PFS \\
\hline II & Erlotinib in combination with chemoradiation in patients with stage IIIA/B NSCLC & Safety \\
\hline
\end{tabular}

${ }^{a}$ www.clinicaltrials.gov

Abbreviations: OS, overall survival; PFS, progression-free survival.

understanding of predictors of benefit with therapy. The next generation of clinical trials will prospectively evaluate the efficacy of EGFR TKIs in clinically or molecularly enriched patient populations. These studies, combined with ongoing advances in technology for detecting EGFR mutations and copy number, will help to further refine the population of patients with NSCLC who are likely to derive the greatest benefit from EGFR TKIs (Table 4).

\section{Disclosure}

The authors report no conflicts of interest in this work.

\section{References}

1. Parkin DM, Bray F, Ferlay J, Pisani P. Estimating the world cancer burden: Globocan 2000. Int J Cancer. 2001;94:153-156.

2. Schrump DS, Altorki NK, Henschke CL, Carter D, Turrisi AT, Gutierrez ME. NSCLC. Cancer: Principles and Practice of Oncology. 7th ed. Philadelphia (PA): Lippincott Williams \& Wilkins; 2005:753-809.

3. Bastin KT, Curley R. NSCLC: Current \& future therapeutic management. Drugs. 1995;49:362-375.

4. D'Addario G, Felip E. Non-small-cell lung cancer: ESMO clinical recommendations for diagnosis, treatment and follow-up. Ann Oncol. 2009:20 Suppl 4:66-70.

5. Felip E, Rosell R. Clinical experience with erlotinib in non-small-cell lung cancer. Drugs Today. 2006;42(3):147-156.

6. Moyer JD, Barbacci EG, Iwata KK, et al. Induction of apoptosis and cell cycle arrest by CP-358,774, an inhibitor of epidermal growth factor receptor tyrosine kinase. Cancer Res. 1997;57:4838-4848.

7. Pollack VA, Savage DM, Baker DA, et al. Inhibition of epidermal growth factor receptor-associated tyrosine phosphorylation in human carcinomas with CP-358,774: Dynamics of receptor inhibition in situ and antitumor effects in athymic mice. J Pharmacol Exp Ther. 1999; 291:739-748.

8. Salomon DS, Brant R, Ciardiello F, et al. Epidermal growth factorrelated peptides and their receptors in human malignances. Crit Rev Oncol Haematol. 1995;19:183-232.

9. Pfizer Inc: CP-358,774 Investigator Brochure 1999. Groton, CT, USA, 1999 Mar.

10. Martin P, Kelly C, Carney D. Epidermal growth factor receptor-targeted agents for lung cancer. Cancer Control. 2006;13(2):129-140.

11. Hidalgo M, Siu LL, Nemunaitis J, et al. Phase I and pharmacologic study of OSI-774, an epidermal growth factor receptor tyrosine kinase inhibitor, in patients with advanced solid malignancies. J Clin Oncol. 2001;19:3267-3279.
12. Milton DT, Azzoli CG, Heelan RT, et al. A phase I/II study of weekly high dose erlotinib in previously treated patients with non-small cell cancer. Cancer. 2006;107:1034-1041.

13. Perez-Soler R, Chachoua A, Hammond LA, et al. Determinants of tumor response and survival with erlotinib in patients with non-small-cell lung cancer. J Clin Oncol. 2004;22:3238-3247.

14. Giaccone G, Gallegos Ruiz M, Le Chevalier T, et al. Erlotinib for frontline treatment of advanced non-small cell lung cancer: A phase II study. Clin Cancer Res. 2006;12:6049-6055.

15. Jackman DM, Yeap BY, Lindeman NI, et al. Phase II clinical trial of chemotherapy-nave patients over 70 years of age treated with erlotinib for advanced non-small-cell lung cancer. J Clin Oncol. 2007; 25:760-766.

16. Hesketh PJ, Chansky K, Wozniak AJ, et al. Erlotinib as initial therapy in patients with advanced non-small cell lung cancer (NSCLC) and a PS of 2: a SWOG Phase II trial (S0341). Proceedings of the American Society Clinical Oncology [Abstract 7536]; 2007, Chicago.

17. Jackman D, Lindeman NI, Lucca J, et al. Phase II study of erlotinib in chemo-nave women with advanced pulmonary adenocarcinoma. Proceedings of the American Society Clinical Oncology [Abstract 7591]; 2007, Chicago.

18. Shepherd FA, Rodrigues Pereira J, Ciuleanu T, et al. Erlotinib in previously treated non-small cell lung cancer. N Engl J Med. 2005;353:123-132.

19. Bezjak A, Tu D, Seymour L, et al. Symptom improvement in lung cancer patients treated with erlotinib: quality of life analysis of the National Cancer Institute of Canada Clinical Trials Group Study BR.21. J Clin Oncol. 2006;24:3831-3837.

20. Herbst RS, Prager D, Hermann R, et al. TRIBUTE: a phase III trial of erlotinib hydrochloride (OSI-774) combined with carboplatin and paclitaxel chemotherapy in advanced non-small-cell lung cancer. J Clin Oncol. 2005;23:5892-5899.

21. Gatzemeier U, Pluzanska A, Szczesna A, et al. Results of a phase III trial of erlotinib (OSI-774) combined with cisplatin and GC chemotherapy in advanced non-small cell lung cancer (NSCLC). J Clin Oncol. 2004;22 Suppl 14:619s.

22. Gatzemeier U, Ardizzoni A, Horwood K. Erlotinib in non-small cell lung cancer (NSCLC): interim safety analysis of the TRUST study. J Clin Oncol. 2007;25 [Abstract 7645].

23. Groen HJM, Barata F, McDermott R, et al. Efficacy of erlotinib in $>4000$ patients with advanced non-small-cell lung cancer (NSCLC): analysis of the European subpopulation of the trust study. ESMO [Abstract 266P], Sweden, 2008 Sep.

24. Tiseo M, Gridelli C, Cascinu S, et al. An expanded access program of erlotinib (Tarceva) in patients with advanced non-small cell lung cancer (NSCLC): data report from Italy. Lung Cancer. 2009;64:199-206.

25. Herbst RS, Johnson DH, Mininberg E, et al. Phase I/II trial evaluating the anti-vascular endothelial growth factor monoclonal antibody bevacizumab in combination with the HER 1/epidermal growth factor receptor tyrosine kinase inhibitor erlotinib for patients with recurrent non-small cell lung cancer. J Clin Oncol. 2005;23:2544-2555. 
26. Fehrenbacher L, O' Neill V, Belani CP, et al. A phase II, multicenter, randomized clinical trial to evaluate the efficacy and safety of bevacizumab in combination with either chemotherapy (docetaxel or pemetrexed) or erlotinib hydrochloride compared with chemotherapy alone for treatment of recurrent or refractory non small cell lung cancer. J Clin Oncol. 2006;24 Suppl 18:379s.

27. Miller VA, O'Connor P, Soh C, et al. A randomized, double-blind, placebo-controlled, phase IIIb trial (ATLAS) comparing bevacizumab therapy with or without erlotinib after completion of chemotherapy with B for first-line treatment of locally advanced, recurrent, or metastatic non-small cell lung cancer (NSCLC). ASCO [Abstract LBA8002];2009.

28. Hainsworth J, Herbst R, A Phase III, multicenter, placebo-controlled, double-blind, randomized clinical trial to evaluate the efficacy of bevacizumab in combination with erlotinib compared with erlotinib alone for treatment of advanced non-small cell lung cancer after failure of standard first-line chemotherapy (BETA). Paper presented at: 2008 Chicago Multidisciplinary Symposium in Thoracic Oncology. 2008 Nov 13-15; Chicago.

29. Thatcher N. First- and second-line treatment of advanced metastatic nonsmall-cell lung cancer: a global view. BMC Proc. 2008; 2 Suppl 2:S3.

30. Clark GM, Zborowski DM, Santabarbara P. Smoking history and epidermal growth factor receptor expression as predictors of survival benefit from erlotinib for patients with non-small-cell lung cancer in the National Cancer Institute of Canada Clinical Trials Group study BR.21. Clin Lung Cancer. 2006;7:389-394.

31. Perez-Soler R, Chachowa A, Hammond LA, et al. Determinants of tumor response and survival with erlotinib in patients with non small cell lung cancer.J Clin Oncol. 2004; 22:3238-3247.

32. Gridelli C, Bareschino MA, Schiettino C. Erlotinib in non small cell lung cancer treatment: current status and future development. Oncologist. 2007;12:840-849.

33. Amador ML, Oppenheimer D, Perea S, et al. An epidermal growth factor receptor intron 1 polymorphism mediates response to epidermal growth factor receptor inhibitors. Cancer Res. 2004;64:9139-9143.

34. Hirsch FR, Varella-Garcia M, Bunn PA, et al. Epidermal growth factor receptor in non-small cell lung carcinomas: correlation between gene copy number and protein expression and impact on prognosis. J Clin Oncol. 2003; 21:3798-3807.

35. Miller VA, Zakowski M, Riely GJ, et al. EGFR mutation and copy number, EGFR protein expression and KRAS mutation as predictors of outcome with erlotinib in bronchioloalveolar cell carcinoma (BAC): results of a prospective phase II trial. J Clin Oncol. Proc ASCO [Abstract 7003]. 2006;24:18S

36. Tsao MS, Sakurada A, Cutz JC, et al. Erlotinib in lung cancermolecular and clinical predictors of outcome. $N$ Engl J Med. 2005; $353: 133-144$.
37. Hirsch FR, Varrella-Garcia M, Bunn PA, et al. Fluorescence in situ hybridization (FISH) subgroup analysis of TRIBUTE, a Phase III trial of erlotinib plus carboplatin and paclitaxel in NSCLC. Proceedings of the American Society Clinical Oncology [Abstract 7570].

38. Shigematsu H, Lin L, Takahashi T, et al. Clinical and biological features associated with epidermal growth factor receptor gene mutations in lung cancers. J Natl Cancer Inst.2005;97:339-346.

39. Johnson BE, Janne PA. Epidermal growth factor receptor mutations in patients with non-small-cell lung cancer. Cancer Res. 2005;65: $7525-7529$

40. Giaccone G. Epidermal growth factor receptor inhibitors in the treatment of non-small-cell lung cancer. J Clin Oncol. 2005;23:3235-3242.

41. Eberhard DA, Johnson BE, Amler LC, et al. Mutations in the epidermal growth factor receptor and in KRAS are predictive and prognostic indicators in patients with non-small-cell lung cancer treated with chemotherapy alone and in combination with erlotinib. J Clin Oncol. 2005;23:5900-5909.

42. Gatzemeier U, Heller A, Foernzler D, et al. Exploratory analyses EGFR, kRAS mutations and other molecular markers in tumors of NSCLC patients (pts) treated with chemotherapy \pm erlotinib (TALENT). Proceedings of the American Society Clinical Oncology [Abstract 7028];2005.

43. Agero AL, Dusza SW, Benvenuto-Andrade C, et al. Dermatologic side effects associated with the epidermal growth factor receptor inhibitors J Am Acad Dermatol. 2006;55:657-670.

44. Busam KJ, Capodieci P, Motzer R, et al. Cutaneous side-effects in cancer patients treated with the epidermal growth factor. BrJ Dermatol. 2001;144:1169-1176.

45. Clark G, Perez-Soler R, Siu L, et al. Rash severity is predictive of increased survival with erlotinib HCI. Proc Am Soc Clin Oncol. 2003;22:196.

46. Perez-Soler R. Can rash associated with HER1/EGFR inhibition be used as a marker of treatment outcome? Oncology. 2003;17:23-28.

47. Ganjoo KN, Wakelee H. Review of erlotinib in the treatment of advanced non-small-cell lung cancer. Biologics. 2007:1(4):335-346.

48. Rosell R, Moran T, Queralt T, et al. Screening for epidermal growth factor receptor mutations in lung cancer. N Engl J Med. 2009;361:958-967.

49. Gridelli C, Butts C, Ciardiello F. An international, multicenter, randomized phase III study of first-line erlotinib followed by second-line cisplatin/gemcitabine versus first-line cisplatin/gemcitabine followed by second-line erlotinib in advanced non-small-cell lung cancer: treatment rationale and protocol dynamics of the TORCH trial. Clin Lung Cancer. July 2008;9(4):235-238.

50. Cappuzzo F, Ciuleanu T, Stelmakh L. Saturn: a double-blind, randomized, phase III study of maintenance erlotinib versus placebo following nonprogression with first-line platinum-based chemotherapy in patients with advanced NSCLC. J Clin Oncol. 2009;27 Suppl; Abstract $8001: 15$ s.
Lung Cancer: Targets and Therapy

\section{Publish your work in this journal}

Lung Cancer: Targets and Therapy is an international, peer-reviewed, open access journal focusing on lung cancer research, identification of therapeutic targets and the optimal use of preventative and integrated treatment interventions to achieve improved outcomes, enhanced survival and quality of life for the cancer patient. Specific topics covered in the journal include:

\section{Dovepress}

Epidemiology, detection and screening; Cellular research and biomarkers; Identification of biotargets and agents with novel mechanisms of action; Optimal clinical use of existing anticancer agents, including combination therapies; Radiation and surgery; Palliative care; Patient adherence, quality of life, satisfaction; Health economic evaluations. 